\title{
Alpine Hydrogeologie
}

\author{
Nico Goldscheider
}

Eingang des Beitrages: 11. 10.2010 / Online veröffentlicht: 13. 1.2011

(C) Springer-Verlag 2011

Hochgebirge spielen eine wichtige Rolle im Wasserhaushalt. Sie erhalten meist mehr Niederschläge als das Umland, während die Evapotranspiration aufgrund der niedrigeren Temperaturen tendenziell geringer ist als im Flachland. Ein bedeutender Teil der Niederschläge fällt als Schnee und wird teils in Gletschern zwischengespeichert, um in wärmeren Perioden wieder in den Wasserkreislauf zu gelangen. Gebirge sind Quellgebiete großer Flüsse und Ausgangspunkt regionaler Grundwasser-Fließsysteme. Die Alpen gelten als Wasserschloss Europas. Dennoch spielt sich hydrogeologische Forschung meist im Flachland ab. Eine Literaturrecherche in hydrogeologischen Fachzeitschriften hat ergeben, dass nur ca. $3 \%$ aller Publikationen einen Bezug zum Gebirge haben; nur ein kleiner Teil davon beschäftigt sich mit alpiner Hydrogeologie im engeren Sinne.

Wie ist diese Diskrepanz zwischen der großen hydrologischen Bedeutung der Hochgebirge und ihrer geringen Beachtung in der hydrogeologischen Literatur zu erklären? Ein Grund liegt vermutlich darin, dass viele hydrogeologische Methoden und Konzepte im Hochgebirge kaum anwendbar sind. In steilem, schwer zugänglichem Gelände lassen sich kaum Beobachtungsbrunnen installieren. Auch das Gesetz von Darcy, Pumpversuche und Grundwassergleichenpläne sind nur eingeschränkt anwendbar. Also machen viele Hydrogeologen lieber einen Bogen ums Hochgebirge, obwohl es dort viele wichtige Fragestellungen zu bearbeiten gäbe:

- Grundwasser im Hochgebirge liefert Trinkwasser für die lokale Bevölkerung und fürs Umland. Die Karstwasserversorgung der Stadt Wien ist ein prominentes Beispiel. In Zeiten des Klimawandels und wachsender Bevölkerung in vielen Teilen der Welt dürfte die Bedeutung der Gebirge für die Wasserversorgung weiter zunehmen.

Prof. Dr. N. Goldscheider

Institut für Angewandte Geowissenschaften, Abt. Hydrogeologie, KIT - Karlsruher Institut für Technologie, Campus Süd, Kaiserstr. 12, 76131 Karlsruhe, Deutschland
- Viele tiefe, hydrothermale Zirkulationssysteme haben ihren Ausgangspunkt im Gebirge; davon zeugen zahlreiche Thermalquellen in den Alpen und ihrem Vorland.

- Grundwasser spielt eine wichtige Rolle bei Bergstürzen und Rutschungen. Durch abschmelzende Gletscher, auftauenden Permafrost und häufigere Starkniederschläge nehmen diese Gefahren zu. Generell macht sich der Klimawandel in den Alpen besonders stark bemerkbar.

- Grundwasserabhängige Ökosysteme werden zunehmend als wertvoll und schützenswert erachtet. Im Hochgebirge ist eine sehr große Vielfalt solcher Biotope anzutreffen.

Auch bei Tunneln im Gebirge spielt Grundwasser eine wichtige Rolle. Dieser Aspekt wurde in den vergangenen Jahren im Zusammenhang mit großen Tunnelprojekten in der Schweiz intensiv bearbeitet. Bei den anderen genannten Themen gibt es dagegen noch viel zu erforschen. Zunächst gilt es, grundsätzliche Konzepte der alpinen Hydrogeologie zu erarbeiten und geeignete Methoden zu entwickeln. Dies fängt bei ganz elementaren Fragen an: Wie mächtig ist die ungesättigte Zone in einem Gebirgsmassiv, wo liegt die Grundwasseroberfläche, falls es eine solche überhaupt gibt, und wie sieht die gesättigte Zone aus? Am weitesten fortgeschritten ist die alpine Hydrogeologie in Karstgebirgen. Aus der Höhlenforschung weiß man, dass die ungesättigte Zone dort teils über $1.000 \mathrm{~m}$ mächtig sein kann. Porenaquifere im Hochgebirge sind einer ständigen Dynamik unterworfen. Durch Massenbewegungen und Hochwässer werden sie neu gebildet, umgelagert oder zerstört. In den großen Alpentälern lassen sich konventionelle hydrogeologische Konzepte und Methoden am ehesten anwenden, aber auch dort gibt es Besonderheiten hinsichtlich der Entstehung, Struktur und Dynamik der Grundwasserleiter. Es wird Zeit, dass sich Hydrogeologen verstärkt mit dem Hochgebirge befassen. Dies erfordert interdisziplinäre Zusammenarbeit mit Hydrologen, Glaziologen, Klimaforschern, Ingenieurgeologen, Bodenkundlern und Ökologen. 